\title{
High-precision tension control method for starting and stopping of optical fiber winding based on trajectory planning
}

\author{
Zhonglin $\mathrm{Guo}^{1, \mathrm{a}}$, Jianhui Zhao ${ }^{1, \mathrm{~b}}$ and $\mathrm{He} \mathrm{Xu}^{1, \mathrm{c}}$ \\ ${ }^{1}$ School of Instument Science and Opto-electronics Engineering, Beihang University, Beijing 100191, \\ China; \\ agzl1992@buaa.edu.cn, bzhaojianhui@buaa.edu.cn, 'xuhe200907@163.com
}

Keywords: optical fibre winding tension control; angular speed trajectory planning; S-shaped acceleration and deceleration curve; starting and stopping of optical fibre winding.

\begin{abstract}
The paper suggests a tension control method based on angular speed trajectory planning to improve tracking angular speed of fiber supply axis and precision of controlling optical fibre tension during the course of starting and stopping of optical fibre winding. First, kinematic trajectory of fibre extraction axis is planned according to five-stage S-shaped acceleration and deceleration curve; then angular speed trajectory of fiber supply axis is planned in a synchronized way according to established tension control system model to realize synchronized planning of active fibre retraction axis and passive fiber supply axis in the course of starting and stopping of optical fibre winding. Test of optical fibre winding acceleration course is conducted on quadripolar optical fibre winder. With this method, the average of tension on optical fibre is $25.0879 \mathrm{~g}$, standard deviation is 0.5218 , overshoot is $7.53 \%$, and tension's frequency within scope of $\pm 1 \mathrm{~g}$ is $94.11 \%$. Compared with integral separating PID control method, this method has high precision in tension control, small overshoot, small tension fluctuation. Applicable to various types of optical fibre winder and spooling equipment, this method has higher theoretical significance and engineering application value.
\end{abstract}

\section{Introduction}

All Optical fibre gyro is mainly used to measure space motion posture of sensitive bearer, and is critical to exert performance of navigation system; optical fibre ring is the core part of optical fibre gyro $^{[1-3]}$. The major factor influencing performance of optical fibre gyro are winding mode of optical fibre, external conditions in winding as well as the tension on optical fibre in winding; To keep constant tension on optical fibre in winding of optical fibre is one of key technologies in winding technology of fibre ${ }^{[4-6]}$.

As quadripolar winding technology of optical fibre is special, and has higher requirements on control precision of tension and winding speed. In the course of optical fibre winding, the fibre retraction axis and fiber supply axis frequently start and stop. If the starting and stopping of motor is not controlled well, this will cause starting jittering and stopping overshoot, which not only influences control precision of tension, but also leads to falling or breakage of optical fibre in severe case; The generally used tension feedback PID control method in industry cannot enable rapid following of fiber supply axis in the course of starting and stopping of optical fibre winding to guarantee constant tension on optical fibre. To cope, the paper suggests a high-precision tension control method based on trajectory planning in the course of starting and stopping of optical fibre.

\section{Modeling of optical fibre winding tension control system}

\section{Structure of tension control system.}

The tension control system structure in this paper uses fibre retraction axis to actively retract fibre, and uses the fibre supply axis to passively supply fibre. The schematic diagram of tension control system of optical fibre winding is as shown in Fig.1. Thereinto 1. Fibre retraction axis realizes unidirectional rotation around spin axis-7 under action of fibre retraction motor. 2. fibre guiding 
pulley 1 realizes guiding for optical fibre between fibre retraction axis and tension pulley. 3 . Tension sensor collects tension signal on optical fibre. 4. Tension pulley applies optical fibre tension on tension sensor. 5. Fibre guiding pulley 2 realizes guiding for optical fibre between tension pulley and fibre supply axis. 6. Fibre supply axis realizes unidirectional rotation around 7-spin axis under action of fibre supply motor.

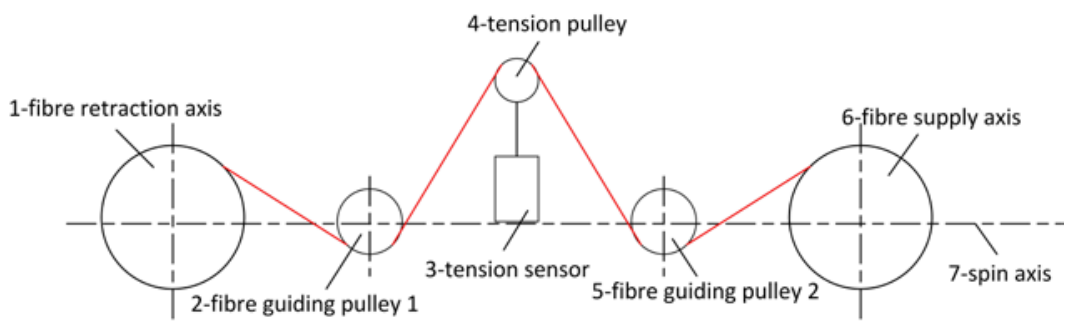

Fig. 1 Schematic diagram of optical fibre winding tension control system

\section{Modeling of optical fibre winding tension control system}

Based on the structure of this system, the tension is generated by the speed difference between fiber retraction axis and fibre supply axis. The control of constant tension in this system is substantially to control linear speed of fibre retraction axis and fiber supply axis to be constant ${ }^{[7]}$. According to Hooke'law, when the optical fiber stress is smaller than tensile limit state, stress $\sigma$ is proportional to strain $\varepsilon$, i.e. $\sigma=E \varepsilon$. Stress $\sigma$ can be expressed as $\sigma=F / S$. Strain $\varepsilon$ can be expressed as $\varepsilon=\Delta L / L$. Where $E$ means elastic modulus of optical fiber, $S$ means cross-sectional area of optical fiber, $\Delta L$ means tensile elongation of optical fiber, $L$ means total length of optical fiber between fiber retraction axis and fiber supply axis, $\Delta F$ means variation of the tension which the optical fiber is subjected to in stretching. Then:

$$
\Delta F=E S \varepsilon=E S(\Delta L / L) .
$$

According to Hook' s law, the variation of tension on optical fiber can be expressed as:

$$
\Delta F=\frac{E S}{L} \int_{0}^{t}\left(v_{r}-v_{p}\right) d t=\frac{E S}{L} \int_{0}^{t}\left(\omega_{r} R_{r}-\omega_{p} R_{p}\right) d t .
$$

Where $\omega_{r}$ is the angular speed of fiber retraction axis and $\omega_{p}$ is angular speed of fiber supply axis; $R_{r}$ and $R_{p}$ are respectively the winding radiuses of fiber retraction axis and fiber supply axis (slowly changing with increase of winding layers of optical fiber), $t$ is the winding time of fiber.

As within certain period during which time the fiber retraction axis and fiber supply axis wind and release a layer of optical fiber, $R_{r}$ and $R_{p}$ are constant value. Assume control and adjustment cycle of motor is $T$. Then within adjustment cycle $T$, the variation of tension on optical fiber is:

$$
\Delta F=\frac{E S}{L}\left(\omega_{r} R_{r}-\omega_{p} R_{p}\right) \times T .
$$

\section{High-precision tension control method based on kinematic trajectory planning}

\section{S-shaped acceleration and deceleration curve}

In the winding course of optical fiber, fiber retraction axis and fiber supply axis frequently start and stop due to reversing and automatic repair of winding defects of optical fiber. As acceleration and deceleration of motor in S-shape curve has no soft shock and has good speed smoothness, thus the fiber retraction motor and fiber supply motor adopt acceleration and deceleration in s-shaped curve. To improve calculation efficiency, the paper simplifies the seven-stage S-shaped acceleration and deceleration into five trajectory stages: accelerating acceleration stage, decelerating acceleration stage, constant speed stage, accelerating deceleration stage, decelerating deceleration stage, as shown in Fig.2. Where $J_{0}$ is constant angular accelerating acceleration, $T_{1}=T_{2}=T_{4}=T_{5}$. 


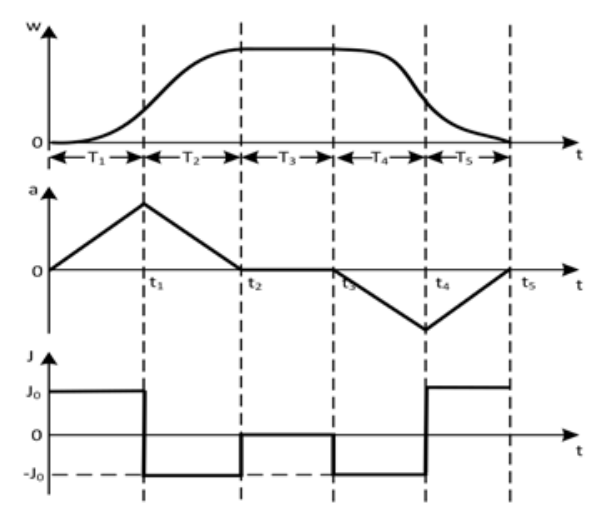

Fig. 2 Five-stage S-shaped acceleration and deceleration curve

\section{Planning of trajectory of starting and stopping course of active fiber retraction axis}

Assume set target angular speed of fiber retraction axis is $\omega_{r_{-} \text {set }}$ (constant), acceleration and deceleration time of S-shaped curve is $T_{\text {var }}$, and calculate the maximum angular acceleration $A_{r_{-} \text {max }_{-} \text {plan }}$ and constant angular accelerating acceleration $J_{r_{-} \text {plan }}$ during the course of acceleration and deceleration of fiber retraction axis according to five-stage S-shaped curve formula:

$$
\begin{aligned}
& A_{r_{-} \text {max }_{-} \text {plan }}=\frac{\omega_{r_{-} \text {set }}}{T_{\mathrm{var}} / 2} . \\
& J_{r_{-} \text {plan }}=\frac{A_{r_{-} \text {max }_{-} \text {plan }}}{T_{\mathrm{var}} / 2}=\frac{4 \omega_{r_{-} \text {set }}}{T_{\mathrm{var}}^{2}} .
\end{aligned}
$$

Assume motor's control and adjustment cycle is $T$, then at $t$, the calculation formulae of angular accelerating acceleration $J_{r}^{(t)}$, angular acceleration $a_{r}^{(t)}$ and angular speed $\omega_{r}^{(t)}$ of fiber retraction axis are respectively as follows. Each adjustment cycle $T$ updates in real time $\omega_{r}^{(t)} 、 a_{r}^{(t)}$ and $J_{r}^{(t)}$, which can realize planning of trajectory of S-shaped acceleration and deceleration of active fiber retraction axis.

$$
\begin{gathered}
J_{r}^{(t)}=\left\{\begin{array}{ll}
J_{r_{-} \text {plan }} & 0 \leq t<t_{1} \\
-J_{r_{-} \text {plan }} & t_{1} \leq t<t_{2} \\
-J_{r_{-} \text {plan }} & t_{3} \leq t<t_{4} \\
J_{r_{-} \text {plan }} & t_{4} \leq t<t_{5}
\end{array} .(6) \quad a_{r}^{(t)}=\left\{\begin{array}{ll}
a_{r}^{(t-T)}+J_{r}^{(t)} T & 0 \leq t<t_{1} \\
a_{r}^{(t-T)}-J_{r}^{(t)} T & t_{1} \leq t<t_{2} \\
0 & t_{2} \leq t<t_{3} \\
a_{r}^{(t-T)}-J_{r}^{(t)} T & t_{3} \leq t<t_{4} \\
a_{r}^{(t-T)}+J_{r}^{(t)} T & t_{4} \leq t<t_{5}
\end{array} .(7)\right.\right. \\
\omega_{r}^{(t)}= \begin{cases}\omega_{r}^{(t-T)}+J_{r}^{(t)} T t-\frac{1}{2} J_{r}^{(t)} T^{2} \\
\omega_{r}^{(t-T)}-J_{r}^{(t)} T t+J_{r}^{(t)} T \times \frac{T_{\mathrm{var}}+T}{2} & t_{1} \leq t<t_{2} \\
\omega_{r}^{(t-T)} & t_{2} \leq t<t_{3} \\
\omega_{r}^{(t-T)}-J_{r}^{(t)} T t+\frac{1}{2} J_{r}^{(t)} T^{2} & t_{3} \leq t<t_{4} \\
\omega_{r}^{(t-T)}+J_{r}^{(t)} T t-J_{r}^{(t)} T \times \frac{T_{\mathrm{var}}+T}{2} & t_{4} \leq t<t_{5}\end{cases}
\end{gathered}
$$

\section{Constant tension algorithm of passive fiber supply axis}

According to above tension control system model, to guarantee constant tension on optical fiber, the linear speeds on active and passive optical fiber axes are required to be synchronized, i.e.:

$$
\omega_{r} R_{r}=\omega_{p} R_{p} \text {. }
$$


As during the period when active and passive optical axes change layer, the winding radius of optical fiber on fiber retraction axis and fiber supply axis is unchanged, thus, the planned instructional angular speed of fiber supply axis $\omega_{p_{-} \text {set }}$ can be expressed as $\omega_{p_{-} \text {set }}=\omega_{r_{-} \text {set }} R_{r_{1}} / R_{p}$.

To keep the tension on optical fiber constant, the angular speed trajectory of planned fiber supply axis should be synchronized with angular speed trajectory of fiber retraction axis, so the time of acceleration and deceleration in S-shaped curve of fiber supply axis is also $T_{\text {var }}$. Calculate the maximum angular acceleration $A_{p_{-} \text {max_ }_{-} \text {plan }}$ in the acceleration and deceleration course of fiber supply axis and constant angular accelerating acceleration $J_{p_{-} \text {plan }}$ according to the formula (4) (5).

Assume motor's adjustment cycle is $T$, then the calculation formulae of angular accelerating acceleration $J_{p}^{(t)}$, angular acceleration $a_{p}^{(t)}$ and angular speed $\omega_{p}^{(t)}$ of fiber retraction axis at $t$ are respectively according to the formular (6) (8). Each adjustment cycle $T$ updates in real time $\omega_{p}^{(t)}$ 、 $a_{p}^{(t)}$ and $J_{p}^{(t)}$, realizing synchronized planning of S-shaped acceleration and deceleration trajectory of fiber supply axis and fiber retraction axis, thereby enabling constant tension control on optical fiber.

\section{Experiment verification}

Test on quadripolar optical fiber winder. Set target tension of optical fiber as $25 \mathrm{~g}$, target retraction speed of fiber retraction axis as $20 \mathrm{r} / \mathrm{min}$, and radius of fiber retraction ring as $16.71 \mathrm{~mm}$, radius of fiber supply ring as $50.45 \mathrm{~mm}$, adjustment time of fiber supply motor as $5 \mathrm{~ms}$. The angular speed of fiber retraction axis is planned according to the five-stage S-shaped acceleration curve, planned angular speed acceleration curve of fiber retraction axis is as shown in Fig.3. The compared results of conventional PID method and the suggested method are shown in Fig.4 and Fig.5.

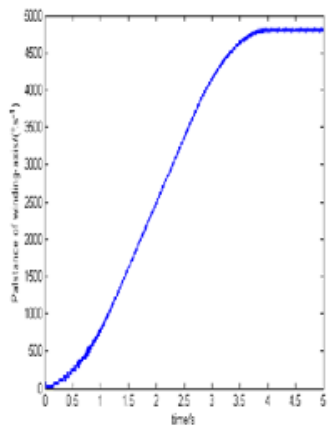

Fig. 3 S-shaped acceleration curve of fiber retraction axis
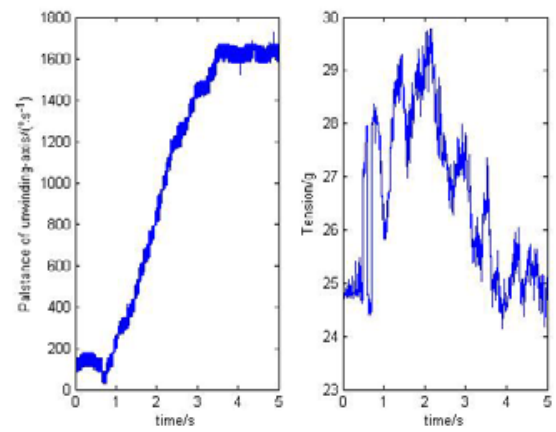

Fig. 4 Angular speed curve and tension change curve of fiber supply axis using conventional PID method
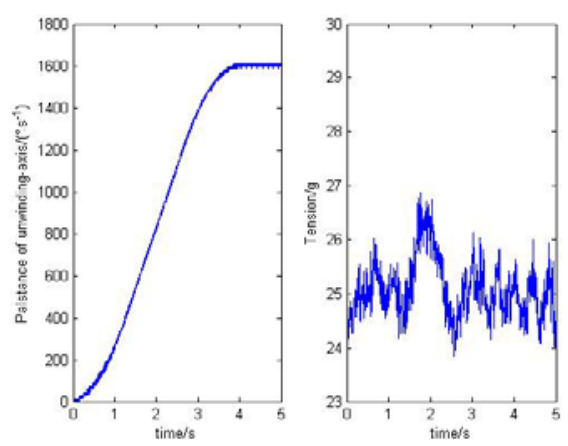

Fig. 5 Angular speed curve and tension change curve of fiber supply axis using tension control method based on trajectory planning

The tension control averages, standard deviations, overshoots, and the probabilities of tension value being controlled at $\pm 1 \mathrm{~g}$ respectively in using integral separating PID control method and the paper's tension control method based on angular speed trajectory planning are calculated as table 1.

Table 1 Comparison of tension indices in using integral separating PID method and the paper's tension control method

\begin{tabular}{ccc}
\hline Tension control indices & Integral separating PID method & The paper’s tension control method \\
\hline Average & 26.4990 & 25.0897 \\
Standard deviation & 1.5336 & 0.5218 \\
Overshoot & $19.18 \%$ & $7.53 \%$ \\
Frequency within $\pm 1 \mathrm{~g}$ & $32.08 \%$ & $94.11 \%$ \\
\hline
\end{tabular}


Figure 4, figure 5 and table 1 show that the integral separating PID control method has lower tension control precision, large overshoot, and large tension fluctuation in the starting course of optical fiber winding; while the paper's tension control method based on angular speed trajectory has higher tension control precision, smaller overshoot, and smaller tension fluctuation, indicating the tension control method based on angular trajectory planning in this paper is far superior to the integral separating PID tension control method.

\section{Summary}

The paper suggests a tension control method based on angular speed trajectory planning. First, kinematic trajectory of fibre extraction axis is planned according to five-stage S-shaped acceleration and deceleration curve; then angular speed trajectory of fiber supply axis is planned in a synchronized way according to established tension control system model to realize synchronized planning of active fibre retraction axis and passive fiber supply axis in the course of starting and stopping of optical fibre winding. Test of optical fibre winding acceleration course is conducted on quadripolar optical fibre winder. Compared with integral separating PID control method, this method has high precision in tension control, small overshoot, small tension fluctuation. Applicable to various types of optical fibre winder and spooling equipment, this method has higher theoretical significance and engineering application value.

\section{Acknowledgments}

This work is supported by the National Natural Science Foundation of China (No.51574012). We sincerely acknowledge the supports.

\section{References}

[1] ZHAO J H, SHU X W, MOU X D. The research of the tension control system of optical fiber coil winding machine [J]. OPTICAL INSTRUMENTS, 2005，27(2): 37-41 . (in Chinese)

[2] LIAO J T. Research on Filament Winding Tension Controlling Based on AC Servo Motor[D]. Wuhan:Wuhan University of Technology,2011.(in Chinese)

[3] GONG J L. Development of Automatic Tension Tontrol System[D]. Hangzhou:Zhejiang University,2006.(in Chinese)

[4] ZHAO L P. Research on Tension Control System for Winding Machine[D]. Tianjin:Tianjin Polytechnic University,2006.(in Chinese)

[5] CHEN X J, LI D, BAI Y. Application of type-II fuzzy neural network to adaptive double axis motion control system[J]. Opt. Precision Eng., 2011,19(7):1643-1650.(in Chinese)

[6] DENG Y T, LI H W, WANG J L. Adaptive sliding mode control for AC servo system based on Kalman filter[J]. Opt. Precision Eng., 2014,22(8):2088-2095. (in Chinese)

[7] ZHANG G, LIU P K, ZHANG B. Design of trajectory tracking controller for precision positioning table driven by linear motor[J]. Opt. Precision Eng., 2013,21(2):371-379.(in Chinese) 